\title{
Adverse Event MedDRA High Level Term
}

National Cancer Institute

\section{Source}

National Cancer Institute. Adverse Event MedDRA High Level Term. NCI Thesaurus.

Code $C 117380$.

A superordinate descriptor for one or more Medical Dictionary for Regulatory Activities (MedDRA) preferred terms (PTs) for the adverse event. 